unter den L-Elementen, die schon eine Reihe von Passagen in der L-Phase hinter sich haben, phagensensible Formen gibt, die während ihrer Vermehrungsphase nicht nur Phagen adsorbieren und lysiert werden, sondern die, wie die Plaque-Bildung zeigt, auch zur Phagensynthese befähigt sind. Bei den phagensensiblen Formen der L-Phase handelt es sich um die Elemente der 3 B-Kolonien, also um diejenigen Elemente des

\section{Additionseffekte bei der Atmung der Hefezelle}

\section{Von F. Tönt und F. Zimmermann}

Bundesanstalt für Materialprüfung Berlin-Dahlem, Abt. Elektrochemische Sonderverfahren

(Z. Naturforschg. 13 b, 472 [1958] ; eingegangen am 21. April 1958)

Die atmende Zelle nimmt in der Zeiteinheit immer die gleiche Menge $\mathrm{O}_{2}$ auf, die graphische Darstellung ihres Atmungsverlaufes ist daher eine Gerade. Dadurch unterscheidet sich die Atmung von dem chemischen Vorgange der Oxydation, deren Intensität durch die jeweils vorhandene $\mathrm{O}_{2}$-Menge bedingt wird.

Die Optimal- oder Vollatmung kann, einmal erreicht, durch weitere Zugaben der gleichen veratembaren Substanz nicht weiter erhöht werden. Ihre Intensität bleibt konstant und hängt nicht von der Art und dem Chemismus des veratmeten Stoffes und auch nicht von der gebotenen $\mathrm{O}_{2}$-Menge, sondern, soviel wir sehen können, allein von der Natur und den Eigenschaften des atmenden Organismus ab.

Die von uns bei den hier zusammengefaßten Versuchen verwendete, aus der laufenden Produktion des Instituts für Gärungsgewerbe, Berlin, entnommene Backhefe ergab unter den gewählten Versuchsbedingungen in Phosphatpufferlösung nach $\mathrm{S}$ örensen mit $p_{\mathrm{H}} 5,4$ bei $20^{\circ} \mathrm{C}$ einen $\mathrm{O}_{2}$-Quotienten $\left(Q_{\mathrm{O}_{2}}\right)$ von 30 bis 35 . Sie veratmet somit in der Stunde je g Trockensubstanz 30 bis $35 \mathrm{~cm}^{3}$ Sauerstoff. Eine, botanisch zur gleichen Art, Saccharomyces cerevisiae gehörige, untergärige Bierhefe, die wir der Schloßbrauerei Berlin verdanken, ergab unter den gleichen Versuchsbedingungen einen $\mathrm{O}_{2}$-Quotienten von nur 9 bis 10 . Bei gleichbleibender Temperatur ist der $Q_{\mathrm{O}_{2}}$ für die Optimalatmung konstant und von der Art der zur Veratmung gebotenen Stoffe nicht oder nur sehr wenig beeinflußt. Die von uns verwendete Backhefe scheint bei zunehmendem Alter Glucose weniger intensiv zu veratmen, die Veratmung von Äthanol dagegen ist vom Alter der atmenden Hefe weniger stark abhängig.

Durch Messung des $\mathrm{O}_{2}$-Verbrauches einer Hefesuspension nach der elektrochemischen Meßmethode von $\mathrm{T} \ddot{\mathrm{d} t}$ gelingt es, auch sehr geringe Differenzen im Atmungsverlauf festzustellen, da es keine prinzipiellen Schwierigkeiten bereitet, den $\mathrm{O}_{2}$-Quotienten für Zeitintervalle von nur zwei Sek. zu bestimmen. Bei den meisten der hier behandelten Versuche begnügten wir uns mit der Feststellung des $Q_{\mathrm{O}_{2}}$ für Zeitspannen von 15 Sekunden.

Vollatmung der von uns studierten Hefe tritt ein, wenn die Suspension $0,018 \%$ oder $180 \mathrm{mg} / l$ Glucose ent-
L-Zyklus, die nach Ausschaltung der Penicillinwirkung zur schnellen Rückbildung in die Bakterienform fähig sind und bei denen eine Ähnlichkeit mit den Normalformen bezüglich der Ausstattung der Zellbegrenzung ja auch zu erwarten ist.

${ }^{6}$ L. Dienes, J. Bacteriol. 57, 529 [1949].

7 W. Harte, Schweiz. Z. Pathol. Bakteriol. 19, 26 [1956].

hält. Durch Zugabe höherer Mengen läßt sich der erreichte $Q_{\mathrm{O}_{2}}$ nicht erhöhen, wohl aber die Dauer der Optimalatmung verlängern. Mit Äthanol in Mengen von $40 \mathrm{mg} / l$ erreicht man die gleiche Optimalatmung wie mit Glucose oder mit 750 bis $1000 \mathrm{mg} / l$ Milchsäure.

Wesentlich scheint nun unsere Feststellung, daß bei Zugabe von zur Vollatmung führenden Mengen von Glucose und Äthanol, einzeln oder auch gemischt, unabhängig von der Reihenfolge der Zugabe eine Verdoppelung der Intensität der Optimalatmung eintritt. Diese quantitative Addition ist kaum anders zu deuten, als daß die Veratmung von Glucose und Äthanol in zwei verschiedenen, nebeneinander und voneinander unabhängig verlaufenden Atmungsvorgängen erfolgt. Fructose, die sich in ihren biologischen Wirkungen mehrfach von Glucose unterscheidet, zeigt insofern ein anderes Verhalten, als in sauren Suspensionen bei $p_{H} 5,4$ der Additionseffekt ausblieb, in dem gleichen Phosphatpuffer bei $p_{\mathrm{H}} 7,5$ aber eintrat. Eine Addition der Optimalatmung von Glucose und Fructose konnte in keinem Falle beobachtet werden, so daß angenommen werden muß, daß beide Zucker im gleichen Atmungsvorgang veratmet werden.

Fügt man zu einer mit Glucose optimal atmenden Hefesuspension Äthanol hinzu, so stellt sich die erhöhte Atmungsintensität in weniger als 15 Sek. ein. Kehrt man die Reihenfolge um und fügt die Glucose der Äthanol veratmenden Suspension zu, dann tritt in den ersten 15 Sek. überhaupt keine Änderung der Atmungsintensität ein. Erst nach 45 Sek. ist die der erfolgten Addition entsprechende verdoppelte Intensität erreicht.

Durch Milchsäure läßt sich die Intensität der Atmung in gleicher Weise zu der durch Glucose und der durch Äthanol bedingten optimalen Intensität addieren und damit verdoppeln. Damit ist nachgewiesen, daß die Veratmung von Milchsäure in einem dritten Atmungsvorgang und unabhängig von der Veratmung von Glucose und von Äthanol erfolgt.

Versuche, alle drei Effekte zu addieren, also in einem Versuche hintereinander Zucker, Alkohol und Milchsäure der suspendierten Hefe zur Veratmung anzubieten und so die optimale Intensität zu verdreifachen, ergaben stark differierende Ergebnisse. Die Reihenfolgen Milchsäure-Glucose-Äthanol und Milchsäure-Äthanol-Glucose ergaben keine wesentliche Addition, welche in den Reihenfolgen Glucose-Milchsäure-Äthanol, ÄthanolMilchsäure-Glucose und besonders Glucose-ÄthanolMilchsäure, allerdings nicht im erwarteten Umfang, erzielt wurde. Da der hier beschriebene Effekt Wege weist, die Atmungsintensität der lebenden Zelle ganz wesentlich zu erhöhen, wird es wohl lohnend sein, weitere systematische Untersuchungen vorzunehmen. 


\section{Monogene Fortpflanzung bei der Fliege Chrysomyia albiceps}

\section{Von F.-H. UlLeRICH}

Max-Planck-Institut für Meeresbiologie, Wilhelmshaven, Abt. H. BAUER

(Z. Naturforschg. 13 b, 473-474 [1958]; eingegangen am 25. Mai 1958)

Monogenie - die Erzeugung rein weiblicher oder rein männlicher Nachkommenschaften bei bisexueller Fortpflanzung - ist bei Insekten nicht häufig. Unter den Dipteren ist sie außer bei den genauer untersuchten Trauermücken (Sciaridae) ${ }^{1}$ nur noch von der tropischen Schmeißfliege (Calliphoride) Chrysomyia rufifacies Macq. $\left(=\right.$ C. albiceps Wied. $\left.{ }^{2}\right)$ bekannt ${ }^{3}$. Während bei den Sciariden die cytologisch-genetische Grundlage der Monogenie aufgeklärt ist, liegen für Chrysomyia nur die Angaben vor, daß Arrhenogenie (Männchenerzeugung), bzw. Thelygenie (Weibchenerzeugung) Eigenschaften der einzelnen $ᄋ \circ$ sind und $\mathrm{da} ß$ die $\hat{\delta} \hat{o}$ keinen Einfluß auf das Geschlecht der Nachkommen haben ${ }^{3}$.

Diese Angaben konnten in umfangreichen Zuchtversuchen mit C.albiceps ${ }^{4}$ bestätigt werden. Alle 우 bringen rein unisexuelle Nachkommenschaften hervor; aus allen (bis zu 7) Folgegelegen eines $q$ entstehen nur Fliegen des gleichen Geschlechts, gleichgültig ob die 우 in Einzel- oder Massenzuchten mit 1 oder mehreren $\hat{o} \hat{o}$ zusammen waren.

In weiblichen Geschwisterschaften treten arrhenogene und thelygene $ᄋ+$ ungefähr im Verhältnis 1:1 auf. In bisher 29 Einzelzuchten wurden insgesamt 1242 우 geprüft; davon waren 608 thelygen und 634 arrhenogen $(P$ für $1: 1=0,46)$. In 2 weiteren Versuchen wurde eine stärkere Verschiebung zugunsten der arrhenogenen 우 beobachtet (15:35 und $3: 17 ; P$ für $1: 1=0,005$ bzw. $<0,0027)$. Ob sich hierin Zufall, modifizierende Außenfaktoren oder Modifikationsgene ausdrücken, wird noch geprüft.

Das 1:1-Verhältnis der beiden -Sorten läßt vermuten, daß wie bei den Sciariden ${ }^{1}$ ein Homo-Heterozygotie-Mechanismus im $q$ für die Monogenie verantwortlich ist, wobei eine o+-Sorte heterozygot für einen dominanten Faktor, die andere homozygot für das rezessive Allel ist. Der sonst bei Calliphoriden vorhandene Geschlechtschromosomen-Apparat mit X-YHeterozygotie der $\hat{\delta} \hat{o} \mathrm{mu}$ dabei ausgeschaltet sein. Bei der Beschränkung der Monogenie auf einen kleinen Verwandtschaftskreis (vielleicht nur eine Art) der Calliphoriden war nicht anzunehmen, daß ein so komplizierter Mechanismus wie bei den Sciariden entwikkelt worden ist. Das hat sich bestätigt.

C. albiceps besitzt wie die übrigen Calliphoriden ${ }^{5}$ $2 n=12$ Chromosomen. In der Metaphase I liegen neben 5 großen V-förmigen Bivalenten 1 Paar kleiner

1 C. W. MEtz, Amer. Naturalist 72, 485 [1938].

2 F. Zumpt, Calliphorinae: E. Lindner, Die Fliegen der palaearktischen Region, Teil 64 i, Stuttgart 1956.

3 D. N. Roy u. L. B. Siddons, Parasitology 31, 442 [1939].
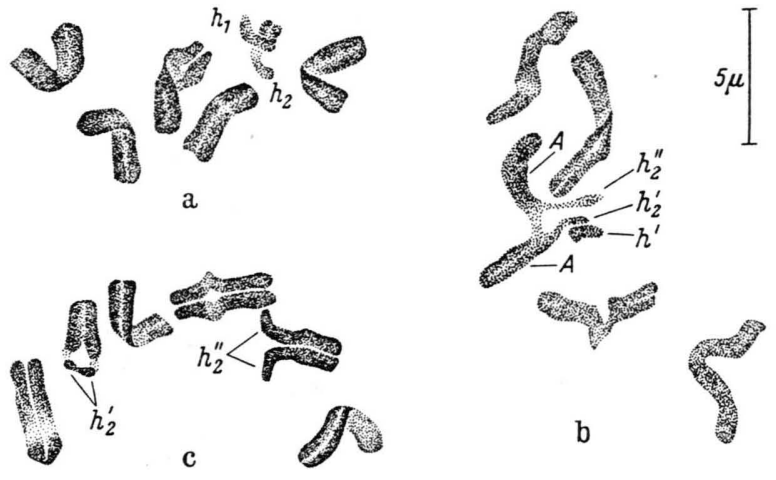

Abb. $1 a-c$. Chromosomenbestände in Spermatocyten I von Chrysomyia albiceps. a Normaler Chromosomenbestand, 5 achiasmatische autosomale Bivalente und ein achiasmatisches Bivalent aus den kleinen Chromosomen $\left(h_{1}, h_{2}\right)$, die nur in einem terminalen Abschnitt gepaart sind. $b$ Heterozygote reziproke Translokation $(\mathrm{T} /+)$ zwischen einem Autosom (A) und einem kleinen Chromosom $\left(\mathrm{h}_{2}\right)$. Die Bruchstellen lagen im Autosom nahe am Kinetochor und im kleinen Chromosom nahe der Mitte. Das nicht-mutierte kleine Chromosom $\left(\mathrm{h}_{1}\right)$ ist mit einem der translozierten Abschnitte $\left(h_{2}{ }^{\prime}\right)$ seines Homologen gepaart (in der Abb. $1 \mathrm{~b}$ ist $h^{\prime}=h_{1}$ ). c Für die gleiche Translokation homozygoter Chromosomenbestand. Orcein-Essigsäure-Milchsäure.

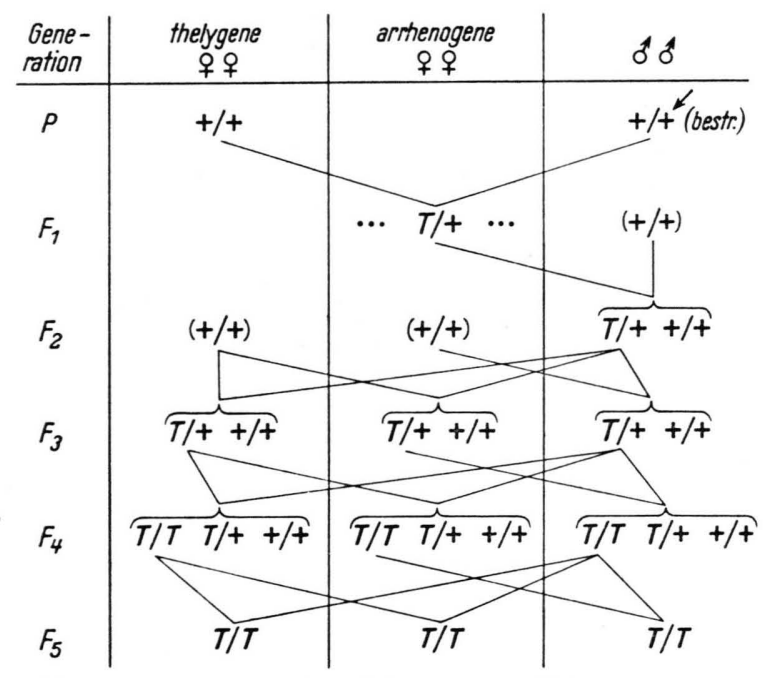

Abb. 2. Erbgang der Translokation aus Abb. 1. T=Translokation, bestr. $=$ röntgenbestrahlt, $(+/+)=$ Tiere aus normalen Stammzuchten.

heterochromatischer Chromosomen vor, die morphologisch den Geschlechtschromosomen von Calliphora entsprechen, aber in beiden Geschlechtern gleich sind (Abb. 1 a). Strukturelle Anzeichen für Heterogametie

4 Material aus Südafrika. Herr Dr. F. Zuмpt, Johannisburg, stellte liebenswürdigerweise Herrn Prof. Bauer lebende Puppen als Ausgangstiere zur Verfügung.

5 C. W. Metz, J. exp. Zool. 21, 213 [1916]; W. Keuneke, Z. Zellenlehre 1, 357 [1924]; A. Naville, Z. Zellforsch. 16, 440 [1932]. 